\title{
PRODUÇÃo DE MATÉRIA SECA E DE GRÃOS POR PLANTAS DE FEIJOEIRO (Phaseolus vulgaris L.) CULTIVADAS EM SOLOS TRATADOS COM METAIS PESADOS
}

\author{
Alexandre Vitor Santana de Carvalho*, Ruy Carvalho e Celeste Maria Patto de Abreu \\ Departamento de Quimica, Universidade Federal de Lavras, 37200-000 Lavras - MG, Brasil \\ Antônio Eduardo Furtini Neto \\ Departamento de Ciência do Solo, Universidade Federal de Lavras, 37200-000 Lavras - MG, Brasil
}

Recebido em 18/5/06; aceito em 11/10/07; publicado na web em 2/4/08

\begin{abstract}
PRODUCTION OF DRY MATERIAL AND GRAINS BY BEAN PLANTS (Phaseolus vulgaris L. ) CULTIVATED IN SOILS TREATED WITH HEAVY METALS. This work aimed at evaluating the contents of extractable $\mathrm{Cd}, \mathrm{Pb}, \mathrm{Zn}$ and $\mathrm{Cu}$ with a solution of DTPA (diethyleneaminopentacetic acid) and to relate them with the production of dry material and grain production of bean plants under two conditions of experimentally contaminated soil materials with $\mathrm{Cd}, \mathrm{Pb}, \mathrm{Cu}$ and $\mathrm{Zn}$ : (i) samples of distrofic red latosol and (ii) humic yellow-red latosol. A decrease in the yield of dry matter was observed for all treatments, if compared with the zero-dose control sample; the grain yield was affected for the soil samples treated with $\mathrm{Cd}$ and $\mathrm{Cu}$ but only slightly for those treated with $\mathrm{Pb}$ and $\mathrm{Zn}$.
\end{abstract}

Keywords: extractor; dry material; heavy metals.

\section{INTRODUÇÃO}

O solo é um componente muito específico da biosfera, pois além de ser um depósito geoquímico de metais contaminantes controla também o transporte das substâncias para a atmosfera, a hidrosfera e a biota.

A contaminação dos solos, decorrente das altas concentrações de metais pesados, comumente ocorre onde há grande concentração de indústrias, que destinam seus resíduos a soterramentos e sedimentação. ${ }^{1}$ Esses sedimentos geram grande preocupação porque agem como acumuladores e transportadores de possíveis fontes de contaminação e se tornam importantes indicadores de poluição ambiental. ${ }^{2-4}$ Os metais pesados acumulam-se freqüentemente na camada superficial do solo $(0-20 \mathrm{~cm})$, também denominada camada agricultável dos solos, tornando-se assim acessíveis às raízes das plantas e, por consequiência, acumuláveis em algumas espécies de organismos vivos. Metais pesados podem persistir no solo por causa da baixa mobilidade, ou serem absorvidos pelas plantas e serem incorporados na cadeia trófica. ${ }^{5}$ A mobilidade desses elementos depende, fundamentalmente, das reações químicas de adsorção e dessorção que ocorrem entre os metais e os componentes sólidos do sistema mineral. As reações são influenciadas por vários fatores, merecendo destaque a presença de ligantes orgânicos e inorgânicos e a CTC (capacidade de troca de cátions) dos solos. ${ }^{6} \mathrm{~A}$ disponibilidade de micronutrientes para as plantas, incluindo a de metais pesados, é influenciada por muitos fatores, particularmente pelo $\mathrm{pH}^{7}$ ? $^{\mathrm{O}}$ efeito da acidez tem implicações importantes na retenção, na mobilidade e na distribuição de metais, principalmente em solos contaminados. ${ }^{8}$ Condições ácidas (pH 4 a 5), por exemplo, são comuns na classe dos solos muito intemperizados, pobres em sílica, como os Latossolos, que ocorre em grandes áreas do território brasileiro e são amplamente utilizados na produção agrícola. ${ }^{9}$ Alguns metais, como $\mathrm{Cu}, \mathrm{Zn}, \mathrm{Mn}$, e Fe são considerados essenciais ao seres vivos quando em pequenas concentrações e como tais denominados micronutrientes. ${ }^{10}$ Com relação aos elementos $\mathrm{Bi}, \mathrm{Cd}, \mathrm{Cr}, \mathrm{Hg}, \mathrm{Ni}, \mathrm{Pb}$ e Sb são considerados não-essenciais e até mesmo tóxicos, dependendo da concentração, merecendo destaque os metais $\mathrm{Cd}, \mathrm{Pb}, \mathrm{Cu}$ e $\mathrm{Zn}$,

*e-mail: avscarvalho@bol.com.br que podem representar toxicidade e ser bioacumulados. ${ }^{11}$ Os metais pesados, como $\mathrm{Hg}$, $\mathrm{Pb}$ e $\mathrm{Cd}$, são perigosos nas suas formas catiônicas se ligados a cadeias curtas de átomos de carbono. ${ }^{12}$ Percebe-se que o termo "metais pesados" engloba elementos não metálicos em que se incluem As, At, e Sb, pela elevada densidade e potencial de toxidez. ${ }^{13}$ Esses metais pesados no solo provêm do intemperismo das rochas de origem do solo, principalmente aquelas ricas em sulfetos, óxidos, silicatos, fosfatos e carbonatos, ${ }^{14}$ por ações antropogênicas, por deposição atmosférica ou da aplicação de agrotóxicos, resíduos orgânicos ou inorgânicos urbanos industriais, lodo de esgoto, fertilizantes ou corretivos. $^{15,16}$

Os teores de metais pesados presentes em solos e disponíveis às plantas são fundamentais na avaliação do risco de entrada desses elementos potencialmente tóxicos na cadeia alimentar. ${ }^{17}$

Para avaliar a disponibilidade de metais pesados, têm sido utilizados métodos de extrações simples, tais como sais neutros, ácidos, bases e agentes quelantes ou complexantes. ${ }^{18}$ Vários outros extratores vêm sendo utilizados para a determinação dos teores de metais pesados em solo. Merecem destaque o Mehlich-1, uma so-

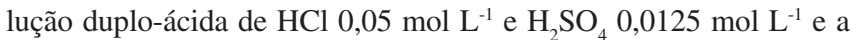
solução DTPA-TEA (ácido dietilenotriaminopentacéticotrietanolamina) ${ }^{19}$ um agente quelatizante..$^{20}$

A solução com DTPA é utilizada para extrair predominantemente as formas lábeis de metais pesados, e é adotada como método oficial dos laboratórios de análise de solo do estado de São Paulo. O princípio de atuação do DTPA é fundamentado em sua capacidade de quelatizar de forma muito estável os íons metálicos da solução do solo. ${ }^{18}$

Há necessidade de se ampliar as pesquisas sobre a produção vegetal, principalmente de grãos, por plantas cultivadas em solos contaminados por metais pesados, especialmente $\mathrm{Cd}$ e $\mathrm{Pb}$. Assim, este trabalho objetivou avaliar os teores de $\mathrm{Cd}, \mathrm{Pb}, \mathrm{Cu}$ e $\mathrm{Zn}$, solúveis em solução de DTPA em amostras de um Latossolo Vermelho Distrófico - $\mathrm{LV}_{\mathrm{d}}$ e de um Latossolo Vermelho-Amarelo Húmico $\mathrm{LVA}_{\mathrm{h}}$, um complexante de uso rotineiro em análises de baixos teores de elementos de transição ( $\mathrm{Fe}, \mathrm{Cu}, \mathrm{Mn}$ e $\mathrm{Zn}$ ), micronutrientes essenciais às plantas em solos agrícolas e, relacionar os teores de $\mathrm{Cd}, \mathrm{Pb}, \mathrm{Cu}$ e $\mathrm{Zn}$ à produção de matéria seca e de grãos por plantas de feijoeiro cultivadas em casa de vegetação. 


\section{PARTE EXPERIMENTAL}

\section{Amostragem e preparação das amostras de solo}

Foram utilizados materiais de dois solos: Latossolo Vermelho Distrófico $\left(\mathrm{LV}_{\mathrm{d}}\right)$ da região de Jaguará, município de São João del Rey, MG e Latossolo Vermelho Amarelo Húmico $\left(\mathrm{LVA}_{\mathrm{h}}\right)$ do município de Barroso, MG. As amostras foram coletadas na camada de 0-20 cm de profundidade. No laboratório, foram secas ao ar e passadas em peneira de $2 \mathrm{~mm}$ de diâmetro. Os atributos químicos, físicos e mineralógicos dos materiais dos solos são apresentados na Tabela 1. O pH foi determinado na relação 1:2,5 (solo:água) por

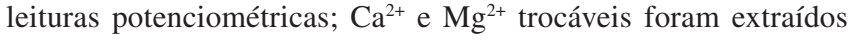
com $\mathrm{KCl} 1 \mathrm{~mol} \mathrm{~L}^{-1}$ e analisados por titulometria; ${ }^{21} \mathrm{H}_{2} \mathrm{PO}_{4}^{-}$e $\mathrm{K}^{+}$ disponíveis foram obtidos com extrator Mehlich-1 e determinados, respectivamente, por colorimetria e fotometria de chama. ${ }^{21,22}$ A acidez potencial $\left(\mathrm{H}^{+}+\mathrm{Al}^{3+}\right)$ foi determinada de forma indireta, por meio de solução Shomacker-Mclean-Pratt (SMP) $\left(\mathrm{CaCl}_{2} \cdot \mathrm{H}_{2} \mathrm{O}\right.$ $+\mathrm{K}_{2} \mathrm{CrO}_{4}+\mathrm{CaC}_{2} \mathrm{O}_{4}+$ trietanolamina, corrigido a $\left.\mathrm{pH} 7,5\right) .{ }^{23}$

\section{Experimentos}

Os experimentos foram conduzidos nos laboratórios dos De-

Tabela 1. Atributos químicos, físicos e mineralógicos dos solos utilizados nos experimentos

\begin{tabular}{|c|c|c|}
\hline Atributos & $\mathrm{LV}_{\mathrm{d}}$ & $\mathrm{LVA}_{\mathrm{h}}$ \\
\hline pH em água & 6,1 & 5,4 \\
\hline $\mathrm{P}_{\text {Mehlich-1 }}\left(\mathrm{mg} \mathrm{dm}^{-3}\right)$ & 0,4 & 2,3 \\
\hline $\mathrm{P}_{\mathrm{rem}}\left(\mathrm{mg} \mathrm{dm}^{-3}\right)$ & 1,3 & 6,5 \\
\hline $\mathrm{K}\left(\mathrm{mg} \mathrm{dm}^{-3}\right)$ & 11,0 & 19,0 \\
\hline $\mathrm{Ca}^{2+}\left(\mathrm{cmol}_{\mathrm{c}} \mathrm{dm}^{-3}\right)$ & 0,5 & 1,2 \\
\hline $\mathrm{Mg}^{2+}\left(\mathrm{cmol}_{\mathrm{c}} \mathrm{dm}^{-3}\right)$ & 0,2 & 0,2 \\
\hline $\mathrm{Al}^{3+}\left(\mathrm{cmol}_{\mathrm{c}} \mathrm{dm}^{-3}\right)$ & 0,0 & 1,2 \\
\hline $\mathrm{H}+\mathrm{Al}\left(\mathrm{cmol}_{\mathrm{c}} \mathrm{dm}^{-3}\right)$ & 1,7 & 7,9 \\
\hline $\mathrm{SB}\left(\mathrm{cmol}_{\mathrm{c}} \mathrm{dm}^{-3}\right)$ & 0,7 & 1,5 \\
\hline $\mathrm{S}-\mathrm{SO}_{4}^{-2}\left(\mathrm{mg} \mathrm{dm}^{-3}\right)$ & 6,2 & 16,6 \\
\hline $\mathrm{t}\left(\mathrm{cmol}_{\mathrm{c}} \mathrm{dm}^{-3}\right)$ & 0,7 & 2,7 \\
\hline $\mathrm{T}\left(\mathrm{cmol}_{\mathrm{c}} \mathrm{dm}^{-3}\right)$ & 2,4 & 9,4 \\
\hline M \% & 0,0 & 45,0 \\
\hline $\mathrm{V} \%$ & 30,0 & 15,5 \\
\hline MO (dag kg $\left.{ }^{-1}\right)$ & 2,5 & 3,1 \\
\hline $\mathrm{B}\left(\mathrm{mg} \mathrm{L}^{-1}\right)$ & 0,1 & 0,2 \\
\hline $\mathrm{Cd}_{\text {DTPA }}\left(\mathrm{mg} \mathrm{dm}^{-3}\right)$ & 0,01 & 0,01 \\
\hline $\mathrm{Pb}_{\text {DTPA }}\left(\mathrm{mg} \mathrm{dm}^{-3}\right)$ & 0,5 & 1,8 \\
\hline $\mathrm{Zn}_{\mathrm{DTPA}}\left(\mathrm{mg} \mathrm{dm} \mathrm{m}^{-3}\right)$ & 0,12 & 0,33 \\
\hline $\mathrm{Cu}_{\text {DTPA }}\left(\mathrm{mg} \mathrm{dm}^{-3}\right)$ & 0,13 & 0,12 \\
\hline $\mathrm{Mn}_{\text {DTPA }}\left(\mathrm{mg} \mathrm{dm}^{-3}\right)$ & 0,5 & 1,0 \\
\hline $\mathrm{Fe}_{\mathrm{DTPA}}\left(\mathrm{mg} \mathrm{dm}^{-3}\right)$ & 11,5 & 36,1 \\
\hline Areia $\left(\mathrm{g} \mathrm{kg}^{-1}\right)$ & 13,0 & 44,0 \\
\hline Silte $\left(\mathrm{g} \mathrm{kg}^{-1}\right)$ & 20,0 & 7,0 \\
\hline Argila $\left(\mathrm{g} \mathrm{kg}^{-1}\right)$ & 67,0 & 49,0 \\
\hline
\end{tabular}

pH em água-relação 1:2,5; $\mathrm{t}$ = capacidade de troca catiônica efetiva; $\mathrm{T}$ = capacidade de troca catiônica a $\mathrm{pH} 7,0 ; \mathrm{V}=$ ídice de saturação de bases; $\mathrm{m}=$ índice de saturação de alumínio; $\mathrm{MO}=$ matéria orgânica; $\mathrm{H}+\mathrm{Al}$ = extrator SMP (Schomacker MClean Pratt); $\mathrm{S}_{-} \mathrm{SO}_{4}=$ extrator fosfato monocálcico acético, $\mathrm{SB}=$ soma de bases trocáveis; $\mathrm{Ca}-\mathrm{Mg}-\mathrm{Al}=$ extrator $\mathrm{KCl} ; \mathrm{P}$ e $\mathrm{K}$ = extrator mehlich-1; $\mathrm{B}$ = extrator água quente; $\mathrm{P}=$ fósforo remanescente. partamentos de Ciência do Solo e de Quimica e em casa de vegetação do Departamento de Ciência do Solo da Universidade Federal de Lavras.

Cada unidade experimental constou de um frasco de polietileno com capacidade para $4 \mathrm{dm}^{3}$ de solo passado em peneira de $2 \mathrm{~mm}$ de diâmetro. Todos os frascos contendo os materiais foram submetidos a duas incubações com soluções contendo os metais em estudo, por um período total de 60 dias, sob condição de umidade equivalente a $60 \%$ do volume total de poros (VTP). ${ }^{24} \mathrm{Na}$ primeira incubação, as doses dos metais pesados utilizados neste experimento foram as seguintes: $\mathrm{Cd}$ : $0 ; 5 ; 10$ e 20; $\mathrm{Pb}: 0 ; 125 ; 250$ e 500; $\mathrm{Cu}: 0$; 1,5; 4,5 e 13,5; Zn: 0; 6; 18 e $54 \mathrm{mg} \mathrm{dm}^{-3}$. As doses de $\mathrm{Cd}$ e $\mathrm{Pb}$ foram escolhidas levando-se em conta valores capazes de "intoxicar sem eliminar" as plantas durante o cultivo. ${ }^{10}$ Quanto ao $\mathrm{Cu}$ e ao $\mathrm{Zn}$, elementos essenciais à nutrição das plantas, os valores das doses utilizadas foram múltiplos dos teores que devem existir nos solos em geral para garantir adequada nutrição: $1,5 \mathrm{mg} \mathrm{dm}^{-3}$ de $\mathrm{Cu}$ e $6 \mathrm{mg} \mathrm{dm}^{-3}$ de Zn. ${ }^{25}$ Foram utilizados sais p. a. como fontes dos elementos contaminadores: $\mathrm{Cd}\left(\mathrm{NO}_{3}\right)_{2} .4 \mathrm{H}_{2} \mathrm{O} ; \mathrm{Pb}\left(\mathrm{NO}_{3}\right)_{2} ; \mathrm{Cu}\left(\mathrm{NO}_{3}\right)_{2}$. $3 \mathrm{H}_{2} \mathrm{O}$ e $\mathrm{Zn}\left(\mathrm{NO}_{3}\right)_{2}$. $4 \mathrm{H}_{2} \mathrm{O}$. Após a aplicação dos metais, as amostras foram incubadas em sacos de polietileno por um período de 30 dias, com o solo mantido sob nível de umidade equivalente a $60 \%$ do VTP. Os teores dos metais pesados solúveis em solução DTPATEA obtidos após a primeira incubação são apresentados na Tabela 2. Decorridos 30 dias da primeira incubação, foi feita a fertilização básica das unidades experimentais diferencialmente para cada tipo de solo, de acordo com o teor individual de argila (Tabela 1). Para o $\mathrm{LV}_{\mathrm{d}}$ foram aplicados: $80 \mathrm{mg} \mathrm{dm}^{-3} \mathrm{de} \mathrm{N} ; 100 \mathrm{mg} \mathrm{dm}^{-3} \mathrm{de} \mathrm{K}$; $350 \mathrm{mg} \mathrm{dm}^{-3}$ de P; $40 \mathrm{mg} \mathrm{dm}^{-3}$ de S; 0,81 $\mathrm{mg} \mathrm{dm}^{-3}$ de B; 3,6 mg dm ${ }^{3}$ de $\mathrm{Mn}$ e $0,15 \mathrm{mg} \mathrm{dm}^{-3}$ de Mo. Para o LVA foram aplicados: 80 $\mathrm{mg} \mathrm{dm}{ }^{-3}$ de $\mathrm{N} ; 100 \mathrm{mg} \mathrm{dm}^{-3}$ de K; $250 \mathrm{mg} \mathrm{dm}^{-3} \mathrm{de} \mathrm{P} ; 40 \mathrm{mg} \mathrm{dm}^{-3} \mathrm{de}$ $\mathrm{S} ; 0,81 \mathrm{mg} \mathrm{dm}^{-3}$ de B; 3,6 $\mathrm{mg} \mathrm{dm}^{-3}$ de Mn e $0,15 \mathrm{mg} \mathrm{dm}^{-3}$ de Mo. Em ambos os solos foi aplicado calcário dolomítico suficiente para manter em 4:1 a relação $\mathrm{Ca}: \mathrm{Mg}$. Os sais p. a. usados nessa etapa foram: $\mathrm{NH}_{4} \mathrm{NO}_{3} ; \mathrm{H}_{3} \mathrm{PO}_{4} ; \mathrm{K}_{2} \mathrm{SO}_{4} ; \mathrm{H}_{3} \mathrm{BO}_{3} ; \mathrm{MnSO}_{4} . \mathrm{H}_{2} \mathrm{O}$; $\left(\mathrm{NH}_{4}\right)_{6} \mathrm{Mo}_{7} \mathrm{O}_{24} \cdot 4 \mathrm{H}_{2} \mathrm{O}$ e calcário dolomítico calcinado e micropulverizado $\{$ Cão $=35 \% \mathrm{MgO}=14 \%$, PRNT (poder relativo de neutralização total $)=100 \%$. Os cálculos referentes à fertilização básica foram feitos considerando-se $3,7 \mathrm{dm}^{3}$ de amostras de solo. Em seguida foi iniciada a segunda incubação sob condição de umidade equivalente a $60 \%$ do VTP. Os resultados analíticos das amostras compostas dos solos obtidos após a segunda incubação são apresentados na Tabela 3.

Tabela 2. Teores dos metais pesados solúveis em solução DTPATEA obtidos após a primeira incubação

\begin{tabular}{|c|c|c|c|c|c|}
\hline \multicolumn{3}{|c|}{ Teor de Cd $\left(\mathrm{mg} \mathrm{dm}^{-3}\right)$} & \multicolumn{3}{|c|}{ Teor de $\mathrm{Pb}\left(\mathrm{mg} \mathrm{dm}^{-3}\right)$} \\
\hline Dose $\left(\mathrm{mg} \mathrm{dm}^{-3}\right)$ & $\mathrm{LVd}$ & LVAh & Dose $\left(\mathrm{mg} \mathrm{dm}^{-3}\right)$ & $\mathrm{LVd}$ & LVAh \\
\hline 0 & 0,01 & 0,01 & 0 & 0,48 & 1,88 \\
\hline 5 & 1,13 & 1,82 & 125 & 47,04 & 21,34 \\
\hline 10 & 1,79 & 3,78 & 250 & 115,41 & 161,57 \\
\hline 20 & 5,85 & 8,42 & 500 & 221,62 & 207,52 \\
\hline \multicolumn{3}{|c|}{ Teor de $\mathrm{Cu}\left(\mathrm{mg} \mathrm{dm}^{-3}\right)$} & \multicolumn{3}{|c|}{ Teor de $\mathrm{Zn}\left(\mathrm{mg} \mathrm{dm}^{-3}\right)$} \\
\hline Dose $\left(\mathrm{mg} \mathrm{dm}^{-3}\right)$ & $\mathrm{LVd}$ & LVAh & Dose $\left(\mathrm{mg} \mathrm{dm}^{-3}\right)$ & LVd & LVAh \\
\hline 0 & 0,11 & 0,64 & 0 & 0,11 & 0,43 \\
\hline 1,5 & 0,75 & 1,17 & 6 & 1,15 & 1,12 \\
\hline 4,5 & 1,36 & 1,95 & 18 & 3,69 & 3,73 \\
\hline 13,5 & 3,39 & 5,97 & 54 & 11,26 & 14,05 \\
\hline
\end{tabular}




\section{Avaliação experimental}

Ao final do ciclo vegetativo as plantas foram colhidas, separadas em parte aérea, vagens e sistema radicular, secas em estufa
(65-70 $\left.{ }^{\circ} \mathrm{C}\right)$, pesadas e moídas, sendo os teores de $\mathrm{Cu}, \mathrm{Zn}, \mathrm{Pb}$ e $\mathrm{Cd}$ da matéria seca da parte aérea (MSPA) e dos frutos dosados utilizando-se um espectrofotômetro de absorção atômica, Carl Zeiss Jena AAS 4, com chama de gás acetileno e lâmpadas de cátodo

Tabela 3. Atributos químicos e físicos dos solos após a aplicação dos tratamentos e fertilização básica.

\begin{tabular}{|c|c|c|c|c|c|c|c|c|}
\hline & & & ses de $\mathrm{C}$ & cadas $(\mathrm{n}$ & & & & \\
\hline Características & & & & & & & & \\
\hline & 0 & 5 & 10 & 20 & 0 & 5 & 10 & 20 \\
\hline pH em água & 7,2 & 7,0 & 6,9 & 7,2 & 6,1 & 6,2 & 6,3 & 6,3 \\
\hline $\mathrm{P}_{\text {mehlich-1 }}\left(\mathrm{mg} \mathrm{dm}^{-3}\right)$ & 13,6 & 15,4 & 14,5 & 14,5 & 55,5 & 54,0 & 57,0 & 55,5 \\
\hline Prem $\left(\mathrm{mg} \mathrm{dm}^{-3}\right)$ & 2,2 & 2,7 & 2,6 & 2,2 & 13,6 & 12,9 & 13,2 & 12,9 \\
\hline 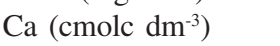 & 3,2 & 3,2 & 3,4 & 3,2 & 2,2 & 1,9 & 2,0 & 2,0 \\
\hline $\left.\mathrm{K}(\mathrm{mg} \mathrm{dm})^{-3}\right)$ & 106,0 & 100,0 & 98,0 & 72,0 & 89,0 & 90,0 & 90,0 & 92,0 \\
\hline $\operatorname{Mg}\left(\right.$ cmolc $\left.\mathrm{dm}^{-3}\right)$ & 0,9 & 0,7 & 0,7 & 0,9 & 0,5 & 0,6 & 0,6 & 0,5 \\
\hline $\mathrm{Al}^{+3}\left(\mathrm{cmolc} \mathrm{dm}^{-3}\right)$ & 0,0 & 0,0 & 0,0 & 0,0 & 0,4 & 0,5 & 0,5 & 0,4 \\
\hline $\mathrm{H}+\mathrm{Al}\left(\mathrm{cmolc} \mathrm{dm}^{-3}\right)$ & 1,0 & 1,1 & 1,2 & 1,1 & 6,3 & 6,3 & 6,5 & 6,3 \\
\hline $\mathrm{SB}\left(\mathrm{cmolc} \mathrm{dm}^{-3}\right)$ & 3,2 & 3,0 & 2,7 & 2,8 & 2,1 & 2,0 & 2,1 & 2,1 \\
\hline $\mathrm{t}\left(\mathrm{cmolc} \mathrm{dm}^{-3}\right)$ & 3,2 & 3,0 & 2,7 & 2,8 & 3,0 & 3,0 & 3,1 & 3,0 \\
\hline $\mathrm{T}\left(\mathrm{cmolc} \mathrm{dm}^{-3}\right)$ & 4,2 & 4,1 & 3,9 & 3,9 & 8,4 & 8,3 & 7,7 & 8,4 \\
\hline $\mathrm{V} \%$ & 76,0 & 72,9 & 68,8 & 71,6 & 25,3 & 24,4 & 27,6 & 25,4 \\
\hline $\mathrm{M} \%$ & 0,0 & 0,0 & 0,0 & 0,0 & 3,0 & 4,0 & 3,0 & 3,0 \\
\hline $\mathrm{MO}\left(\mathrm{g} \mathrm{kg}^{-1}\right)$ & 1,9 & 2,0 & 1,8 & 1,8 & 2,6 & 3,0 & 2,9 & 3,0 \\
\hline $\mathrm{Cd}\left(\mathrm{mg} \mathrm{dm}^{-3}\right)$ & 0,01 & 0,36 & 1,26 & 3,73 & 0,01 & 1,1 & 3,2 & 8,1 \\
\hline & & & ses de $\mathrm{P}$ & cadas $(n$ & & & & \\
\hline Características & & & & & & & & \\
\hline & 0 & 125 & 250 & 500 & 0 & 125 & 250 & 500 \\
\hline pH em água & 7,2 & 7,2 & 7,1 & 6,6 & 6,4 & 6,1 & 6,3 & 6,0 \\
\hline $\mathrm{P}_{\text {mehlich-1 }}\left(\mathrm{mg} \mathrm{dm}^{-3}\right)$ & 13,6 & 14,1 & 14,1 & 15,0 & 55,5 & 57,0 & 65,4 & 60,2 \\
\hline Prem $\left(\mathrm{mg} \mathrm{dm}^{-3}\right)$ & 2,2 & 2,4 & 2,2 & 2,6 & 13,6 & 13,6 & 14,0 & 11,8 \\
\hline $\mathrm{Ca}\left(\mathrm{cmolc} \mathrm{dm}^{-3}\right)$ & 3,2 & 3,2 & 3,0 & 3,2 & 2,1 & 1,9 & 2,0 & 2,0 \\
\hline $\mathrm{K}\left(\mathrm{mg} \mathrm{dm}^{-3}\right)$ & 106,0 & 103,0 & 106,0 & 108,0 & 89,0 & 84,0 & 97,0 & 105,0 \\
\hline $\operatorname{Mg}\left(\mathrm{cmolc} \mathrm{dm}^{-3}\right)$ & 0,9 & 0,7 & 0,7 & 0,9 & 0,5 & 0,5 & 0,4 & 0,5 \\
\hline $\mathrm{Al}^{+3}\left(\mathrm{cmolc} \mathrm{dm}^{-3}\right)$ & 0,0 & 0,0 & 0,0 & 0,0 & 0,4 & 0,5 & 0,5 & 0,4 \\
\hline $\mathrm{H}+\mathrm{Al}\left(\mathrm{cmolc} \mathrm{dm}^{-3}\right)$ & 1,0 & 1,1 & 1,1 & 1,1 & 6,3 & 6,3 & 6,3 & 6,3 \\
\hline $\mathrm{SB}\left(\mathrm{cmolc} \mathrm{dm}^{-3}\right)$ & 3,0 & 3,0 & 3,0 & 3,2 & 2,1 & 2,2 & 2,3 & 2,5 \\
\hline $\mathrm{t}\left(\mathrm{cmolc} \mathrm{dm}^{-3}\right)$ & 3,2 & 3,0 & 3,0 & 3,2 & 3,0 & 3,2 & 3,2 & 3,5 \\
\hline $\mathrm{T}\left(\mathrm{cmolc} \mathrm{dm}^{-3}\right)$ & 4,2 & 4,1 & 4,1 & 4,2 & 8,4 & 8,5 & 8,6 & 8,8 \\
\hline $\mathrm{V} \%$ & 76,0 & 72,9 & 73,0 & 76,0 & 25,3 & 26,2 & 26,3 & 28,2 \\
\hline $\mathrm{M} \%$ & 0,0 & 0,0 & 0,0 & 0,0 & 4,0 & 4,0 & 3,0 & 4,0 \\
\hline $\mathrm{MO}\left(\mathrm{g} \mathrm{kg}^{-1}\right)$ & 1,9 & 2,0 & 1,8 & 2,1 & 2,6 & 3,0 & 3,0 & 2,9 \\
\hline $\mathrm{Pb}\left(\mathrm{mg} \mathrm{dm}^{-3}\right)$ & 0,43 & 63,94 & 184,46 & 311,67 & 2,81 & 58,31 & 222,69 & 322,5 \\
\hline & & & ses de C & cadas $(\mathrm{n}$ & & & & \\
\hline Características & & & & & & & & \\
\hline & 0 & 1,5 & 4,5 & 13,5 & 0 & 1,5 & 4,5 & 13,5 \\
\hline pH em água & 7,2 & 7,3 & 7,3 & 7,2 & 6,2 & 6,1 & 6,3 & 6,2 \\
\hline $\mathrm{P}_{\text {mehlich- } 1}\left(\mathrm{mg} \mathrm{dm}^{-3}\right)$ & 13,6 & 13,6 & 15,4 & 14,1 & 55,5 & 51,1 & 51,1 & 57,0 \\
\hline Prem $\left(\mathrm{mg} \mathrm{dm}^{-3}\right)$ & 2,2 & 2,0 & 2,0 & 2,0 & 13,6 & 13,2 & 12,9 & 14,3 \\
\hline $\mathrm{Ca}\left(\mathrm{cmolc} \mathrm{dm}^{-3}\right)$ & 3,2 & 3,4 & 3,5 & 3,4 & 2,1 & 2,0 & 2,2 & 2,0 \\
\hline $\mathrm{K}\left(\mathrm{mg} \mathrm{dm} \mathrm{m}^{-3}\right)$ & 106,0 & 95,0 & 105,0 & 106,0 & 89,0 & 94,0 & 92,0 & 83,0 \\
\hline $\mathrm{Mg}\left(\mathrm{cmolc} \mathrm{dm}^{-3}\right)$ & 0,9 & 0,8 & 0,9 & 1,0 & 0,5 & 0,5 & 0,6 & 0,6 \\
\hline $\mathrm{Al}^{+3}\left(\mathrm{cmolc} \mathrm{dm}^{-3}\right)$ & 0,0 & 0,0 & 0,0 & 0,0 & 0,4 & 0,5 & 0,5 & 0,4 \\
\hline 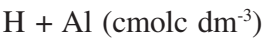 & 1,0 & 1,1 & 1,1 & 1,1 & 6,3 & 6,3 & 6,3 & 6,3 \\
\hline SB $\left(\right.$ cmolc dm $\left.{ }^{-3}\right)$ & 3,0 & 2,9 & 3,2 & 3,2 & 2,1 & 2,1 & 2,1 & 2,1 \\
\hline $\mathrm{t}\left(\mathrm{cmolc} \mathrm{dm}^{-3}\right)$ & 3,2 & 2,9 & 3,2 & 3,2 & 3,0 & 3,1 & 3,0 & 3,1 \\
\hline $\mathrm{T}\left(\mathrm{cmolc} \mathrm{dm}^{-3}\right)$ & 4,2 & 4,0 & 4,3 & 4,3 & 8,4 & 8,4 & 8,4 & 8,4 \\
\hline $\mathrm{V} \%$ & 76,0 & 72,8 & 74,2 & 74,2 & 25,3 & 25,4 & 25,4 & 25,1 \\
\hline $\mathrm{M} \%$ & 0,0 & 0,0 & 0,0 & 0,0 & 3,0 & 4,0 & 4,0 & 3,0 \\
\hline $\mathrm{MO}\left(\mathrm{g} \mathrm{kg}^{-1}\right)$ & 1,9 & 2,2 & 2,1 & 2,2 & 3,0 & 2,9 & 3,0 & 3,0 \\
\hline $\mathrm{Cu}\left(\mathrm{mg} \mathrm{dm}{ }^{-3}\right)$ & 0,12 & 0,25 & 0,47 & 1,61 & 0,15 & 0,26 & 0,54 & 2,38 \\
\hline
\end{tabular}


Tabela 3. continuaçao

\begin{tabular}{|c|c|c|c|c|c|c|c|c|}
\hline & & & ses de & cadas $(1$ & & & & \\
\hline Características & & & & & & & & \\
\hline & 0 & 6 & 18 & 54 & 0 & 6 & 18 & 54 \\
\hline pH em água & 7,2 & 7,4 & 7,4 & 7,2 & 6,1 & 6,2 & 6,2 & 6,3 \\
\hline $\mathrm{P}_{\text {mehlich-1 }}\left(\mathrm{mg} \mathrm{dm}^{-3}\right)$ & 13,6 & 13,6 & 13,6 & 16,4 & 55,5 & 58,6 & 63,6 & 58,6 \\
\hline Prem $\left(\mathrm{mg} \mathrm{dm}^{-3}\right)$ & 2,2 & 2,2 & 2,0 & 2,2 & 13,6 & 14,3 & 15,6 & 13,2 \\
\hline $\mathrm{Ca}\left(\mathrm{cmolc}^{-3}\right)$ & 3,2 & 3,0 & 3,2 & 3,5 & 2,2 & 2,0 & 2,1 & 2,0 \\
\hline $\mathrm{K}\left(\mathrm{mg} \mathrm{dm}^{-3}\right)$ & 106,0 & 95,0 & 103,0 & 108,0 & 89,0 & 103,0 & 97,0 & 98,0 \\
\hline $\mathrm{Mg}\left(\mathrm{cmolc} \mathrm{dm}^{-3}\right)$ & 0,9 & 0,8 & 0,8 & 1,0 & 0,6 & 0,6 & 0,5 & 0,5 \\
\hline $\mathrm{Al}^{+3}\left(\mathrm{cmolc} \mathrm{dm}^{-3}\right)$ & 0,0 & 0,0 & 0,0 & 0,0 & 0,5 & 0,4 & 0,5 & 0,5 \\
\hline $\mathrm{H}+\mathrm{Al}\left(\mathrm{cmolc} \mathrm{dm}^{-3}\right)$ & 1,0 & 1,0 & 1,1 & 1,1 & 6,3 & 6,3 & 6,3 & 6,3 \\
\hline $\mathrm{SB}\left(\mathrm{cmolc} \mathrm{dm}^{-3}\right)$ & 3,0 & 2,9 & 3,0 & 3,2 & 2,1 & 2,2 & 2,2 & 2,3 \\
\hline $\mathrm{t}\left(\mathrm{cmolc} \mathrm{dm}^{-3}\right)$ & 3,2 & 2,9 & 3,0 & 3,2 & 3,0 & 3,1 & 3,1 & 3,2 \\
\hline $\mathrm{T}\left(\mathrm{cmolc} \mathrm{dm}^{-3}\right)$ & 4,2 & 3,9 & 4,1 & 4,3 & 8,4 & 8,5 & 8,4 & 8,6 \\
\hline $\mathrm{V} \%$ & 76,0 & 74,6 & 72,9 & 74,3 & 25,3 & 25,5 & 25,4 & 26,3 \\
\hline $\mathrm{M} \%$ & 0,0 & 0,0 & 0,0 & 0,0 & 3,0 & 3,0 & 4,0 & 3,0 \\
\hline MO (dag kg-1) & 1,9 & 2,2 & 2,1 & 2,0 & 2,6 & 3,0 & 2,9 & 3,0 \\
\hline $\mathrm{Zn}\left(\mathrm{mg} \mathrm{dm}^{-3}\right)$ & 0,13 & 0,67 & 2,84 & 9,55 & 0,33 & 1,32 & 4,01 & 10,18 \\
\hline
\end{tabular}

pH em água-relação $1: 2,5 ; \mathrm{t}=$ capacidade de troca catiônica efetiva; $\mathrm{T}=$ capacidade de troca catiônica a pH 7,0; $\mathrm{V}=$ ídice de saturação de bases; $\mathrm{m}$ = índice de saturação de alumínio; $\mathrm{MO}=$ matéria orgânica; $\mathrm{H}+\mathrm{Al}=$ extrator $\mathrm{SMP}\left(\mathrm{Schomacker} \mathrm{MClean}_{\mathrm{Pratt}}\right) ; \mathrm{S}-\mathrm{SO}_{4}=$ extrator fosfato monocálcico acético, $\mathrm{SB}=$ soma de bases trocáveis; $\mathrm{Ca}-\mathrm{Mg}-\mathrm{Al}=$ extrator $\mathrm{KCl}$; $\mathrm{P}$ e $\mathrm{K}=$ extrator mehlich-1; $\mathrm{B}=$ extrator água quente; $\mathrm{P}$ = fósforo remanescente.

ôco: Cd (228,8 nm, fenda de 0,5); $\mathrm{Pb}(217 \mathrm{~nm}$, fenda de 1,0$) ; \mathrm{Cu}$ $(324,8 \mathrm{~nm}$, fenda de 0,5$)$ e Zn $(213,9 \mathrm{~nm}$, fenda de 0,5$)$. Nesta pesquisa as produções de MSPA e de grãos foram comparadas com os teores de $\mathrm{Cd}, \mathrm{Pb}, \mathrm{Cu}$ e $\mathrm{Zn}$ presentes dos solos após a segunda incubação, extraídos pela solução DTPA-TEA, utilizando-se uma relação solo-extrator igual a 1:5. ${ }^{19}$

\section{Extração com DTPA}

Após um período de 30 e 60 dias da aplicação das doses, foram transferidos de cada tratamento $10 \mathrm{~cm}^{3}$ de terra fina seca ao ar (TFSA) para frascos de vidro, juntamente com $50 \mathrm{~mL}$ da solução extratora (DTPA $0,005 \mathrm{~mol} \mathrm{~L}^{-1}+$ trietanolamina $0,1 \mathrm{~mol} \mathrm{~L}^{-1}+$ cloreto de cálcio $0,01 \mathrm{~mol} \mathrm{~L}^{-1}+5 \mathrm{~mL}$ de $\mathrm{HCl}$ e corrigido a $\left.\mathrm{pH} 7,3\right) \cdot{ }^{19}$ Após agitação mecânica (velocidade de $240 \mathrm{rpm}$ ) por $2 \mathrm{~h}$, decantou-se a solução e procedeu-se à análise de $\mathrm{Cd}, \mathrm{Pb}, \mathrm{Cu}$ e $\mathrm{Zn}$, por espectrofotometria de absorção atômica.

\section{Delineamento experimental e estatístico}

$\mathrm{O}$ delineamento experimental utilizado foi o inteiramente casualizado (DIC), arranjado em um esquema fatorial $(2 \times 4)$, sendo dois solos (Latossolo Vermelho distrófico e Latossolo Vermelho-Amarelo-Húmico) e quatro doses para cada metal: Cd: $0 ; 5 ; 10$ e $20 \mathrm{mg} \mathrm{dm}^{-}$ 3; Pb: 0, 125; 250 e 500 mg dm${ }^{-3}$; Cu: 0; 1,5; 4,5 e 13,5 mg dm ${ }^{-3}$ e Zn: $0 ; 6 ; 18$ e $54 \mathrm{mg} \mathrm{dm}^{-3}$, com quatro repetições para cada tratamento. Os resultados de valores médios de quatro repetições foram submetidos à teste de Tukey utilizando-se o programa SANEST. ${ }^{26}$

\section{RESULTADOS E DISCUSSÃO}

\section{Cádmio}

Em relação aos teores de Cd extraídos com DTPA (Figura 1a) ocorreu menor extração no $\mathrm{LV}_{\mathrm{d}}$ foi de $3,72 \mathrm{mg} \mathrm{dm}^{-3}(18,6 \%)$ e no $\mathrm{LVA}_{\mathrm{h}}$ foi de $8,09 \mathrm{mg} \mathrm{dm}^{-3}(40,5 \%)$, considerando a maior dose aplicada que foi de $20 \mathrm{mg} \mathrm{dm}^{-3}$. Os dados obtidos neste estudo parecem coincidir com aqueles do trabalho realizado por Pierangeli et $a l .{ }^{8}$ que avaliaram a adsorção e a dessorção de $\mathrm{Cd}$ em Latossolos brasileiros. Demonstraram que a elevação do $\mathrm{pH}$ resulta em um aumento da capacidade de adsorção do Cd. Pode-se verificar que os valores de $\mathrm{pH}$ da Tabela 3 estão entre 7 e 7,2 para o $\mathrm{LV}_{\mathrm{d}}$, coincidem com os menores teores extraídos (Figura 1a), enquanto para o LVA $_{\mathrm{h}}$, com pH entre 6,1 e 6,3 (Tabela 3), ocorre uma maior extração. Esse fato pode também ser observado em relação ao $\mathrm{pH}$ do solo amostrado que foi de 6,1 para $\mathrm{LV}_{\mathrm{d}}$ e 5,4 para $\mathrm{LVA}_{\mathrm{h}} \mathrm{O} \mathrm{Cd}$ é fracamente adsorvido à matéria orgânica, argilominerais e óxidos de $\mathrm{Fe}$ e $\mathrm{Al}$ em pH inferior a 6,0. ${ }^{6}$ Esse fenômeno pode ser atribuído ao fato das superfícies de troca sofrerem desprotonação com a conseqüiente geração de cargas negativas. ${ }^{27}$ Essa é a dinâmica dos Latossolos, um material cujas superfícies de troca são dependentes de $\mathrm{pH}$. Para o LVA, $\mathrm{pH}$ entre 6,1 e 6,3 (Tabela 3), parece que a matéria orgânica (MO) não influenciou a retenção do $\mathrm{Cd}$ já que houve uma maior extração (Figura 1a).

Com relação à influência da MO na retenção de metais, de modo geral, cabe ressaltar a dificuldade de comparação entre os vários trabalhos existentes na literatura. Isso porque a MO apresenta diversos grupamentos funcionais reativos, isto é, com diferentes capacidades de doar elétrons e como tais, responsáveis pela retenção diferenciada de metais, como é o caso das aminas, iminas, carboxilas, fenóis, álcoois e sulfidrilas. Assim, é razoável admitir que o comportamento da MO na adsorção de metais será distinto quando for estudado em diferentes tipos de solo, com diferentes tipos de MO.

Em relação à produção de matéria seca da parte aérea pelas plantas cultivadas (Figura 1b), observa-se que esse parâmetro foi fortemente afetado a partir da dose $5 \mathrm{mg} \mathrm{dm}^{-3}$.

Comparando-se o tratamento-controle e a dose máxima aplicada do metal, observou-se o seguinte: para $\mathrm{LV}_{\mathrm{d}}$ no tratamento controle houve uma produção de $13,1 \mathrm{~g}$, caindo para $4,4 \mathrm{~g}$ no tratamento que recebeu a dose máxima, ou seja $20 \mathrm{mg} \mathrm{dm}^{-3}$, variando em torno de $65,0 \%$ a queda na produção de MSPA. Para o LVA, o tratamento-controle apresentou uma produção de $13,1 \mathrm{~g}$ enquanto aquele que recebeu a maior dose $\left(20 \mathrm{mg} \mathrm{dm}^{-3}\right)$ caiu para $4,4 \mathrm{~g}$, variando em $66 \%$ a queda de produção (Figura $1 \mathrm{~b}$ ).

Na Figura 1c encontram-se a relação das doses aplicadas e a produção de grãos. Observa-se que há queda na produção de grãos 
Teor extraído

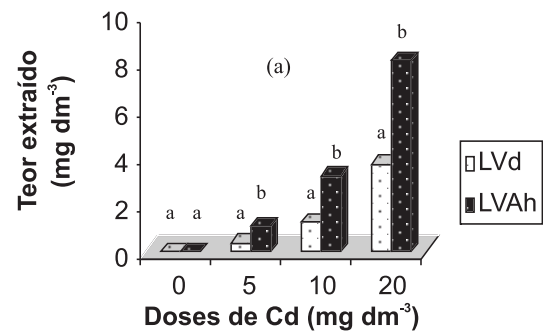

Teor extraído

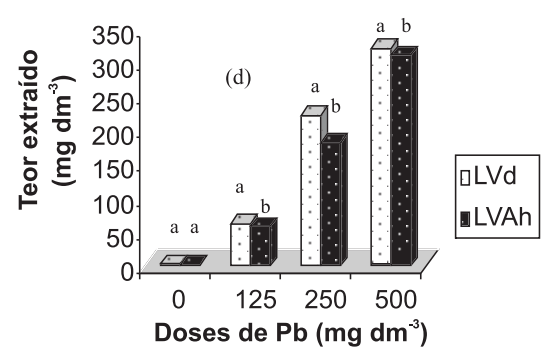

MSPA

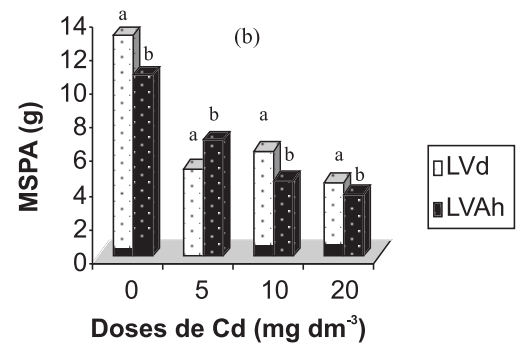

MSPA

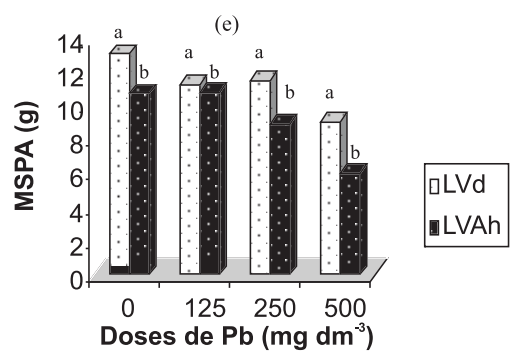

Produção de grãos

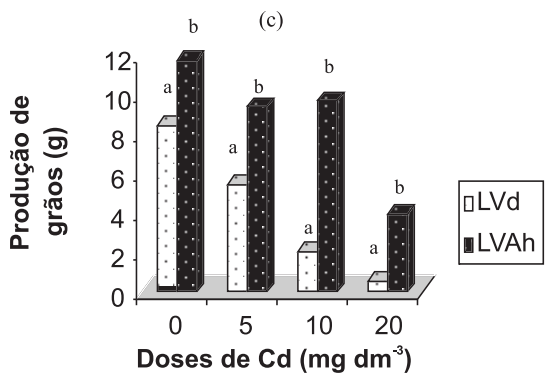

Produção de grãos

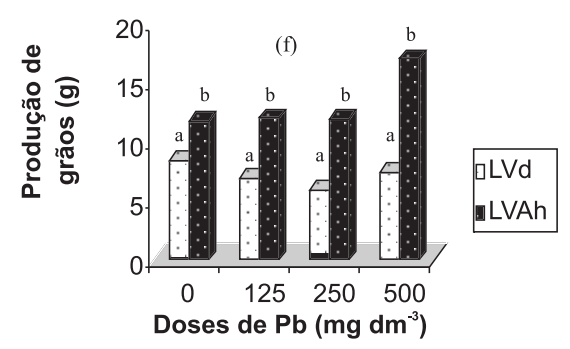

Figura 1. Teores de metais solúveis no extrator DTPA, produção de matéria seca e de grãos por plantas de feijoeiro cultivadas em dois solos, em função das doses de $C d(a, b, c)$ e de $\mathrm{Pb}(d, e, f)$ aplicadas. Médias seguidas pela mesma letra não diferem entre si pelo teste de Tukey em nível de $5 \%$ de probabilidade

à medida que se aumentam as doses aplicadas. No $\mathrm{LV}_{\mathrm{d}}$, considerando as dose 5,10 e $20 \mathrm{mg} \mathrm{dm}^{-3}$, o decréscimo de produção foi de 35,0; 75,0 e 93,0\%, respectivamente. Já no LVA, comparando-se o teor extraído de Cd pelo DTPA (Figura 1a) que foi maior que no $\mathrm{LV}_{\mathrm{d}}$, a produção de grãos decresceu linearmente do tratamentocontrole para a dose de $20 \mathrm{mg} \mathrm{dm}^{-3}$, tendo uma redução de $66,0 \%$. No $L V_{d}$, a queda da produção de grãos comparando os dois extremos das doses foi de 93,0\%. Percebe-se que embora altamente nocivo às plantas nos dois solos, os efeitos do $\mathrm{Cd}$ foram mais evidentes nas plantas cultivadas no material de solo mais pobre em matéria orgânica e mais rico em argila.

A redução de MSPA e de grãos nas plantas contaminadas com $\mathrm{Cd}$ ocorre, provavelmente, em função da exposição de vegetais ao $\mathrm{Cd}$ provocar severas mudanças, por exemplo, na taxa fotossíntetica. ${ }^{28}$

\section{Chumbo}

Os teores de $\mathrm{Pb}$ extraídos pela solução de DTPA apresentaram acréscimos significativos com o aumento da dose de chumbo aplicada (Figura 1d), com pequenas diferenças estatísticas nos dois solos, sendo que para a maior dose aplicada o DTPA extraiu cerca de $64,0 \%$ do $\mathrm{Pb}$ aplicado. Esses resultados parecem demonstrar que a maior parte do metal, independente da dose aplicada, pode estar disponível para as plantas na solução do solo, apesar do $\mathrm{Pb}$ ser considerado um dos metais pesados menos móveis no solo. ${ }^{29}$

Em geral, o pH deve exceder 6,5 a fim de minimizar a mobilidade e a toxicidade de metais pesados. ${ }^{8}$ Tanto no $\mathrm{LV}_{\mathrm{d}}$ amostrado (Tabela 1) quanto naquele que recebeu fertilização básica (Tabela 3) o pH oscilou de 6,1 a 7,2, tendendo à neutralidade ou baixa basicidade. Nesse contexto, o pH também parece justificar a menor mobilidade (ou menor disponibilidade) do $\mathrm{Pb}$ em solução no $\mathrm{LV}_{\mathrm{d}}$ que no $\mathrm{LVA}_{\mathrm{h}}$.

Uma análise mais detalhada da Tabela 3 confirma as observações anteriores: à medida que se aumenta a dose de $\mathrm{Pb}$ aplicada o $\mathrm{pH}$ decresce, tanto no $\mathrm{LV}_{\mathrm{d}}$ quanto no $\mathrm{LVA}_{\mathrm{h}}$, com $\mathrm{pH}$ mais baixo no $\mathrm{LVA}_{\mathrm{h}}$. No $\mathrm{LV}_{\mathrm{d}}$ a grande quantidade de $\mathrm{Pb}$ extraído pode ser devido ao fato deste solo apresentar incremento de CTC. Afinal, em "condições de vaso", onde não existem as perdas por percolação, não só o $\mathrm{Pb}$, mas qualquer elemento permanece mais na solução do solo, estando, desse modo, mais disponível às plantas. ${ }^{30}$

Para a produção de MSPA no $\operatorname{LV}_{\mathrm{d}}$ (Figura 1e) houve queda de matéria seca à medida que se aumentou a dose do metal aplicada, tendo uma variação de $31,2 \%$ entre o tratamento-controle e a maior dose aplicada (500 mg dm $\mathrm{m}^{-3}$ ). Para o $\mathrm{LVA}_{\mathrm{h}}$ as doses 125 e $250 \mathrm{mg}$ $\mathrm{dm}^{-3}$ parecem ter afetado pouco a produção de MSPA, enquanto a dose de $500 \mathrm{mg} \mathrm{dm}^{-3}$ provocou decréscimo de $31,0 \%$ na produção de MSPA.

Conforme a Figura 1f, que mostra o comportamento da produção de grãos, o efeito do $\mathrm{Pb}$ sobre esse parâmetro não foi tão significativo: na dose $125 \mathrm{mg} \mathrm{dm}^{-3}$ houve uma queda de $18,7 \%$ na produção para $\mathrm{LV}_{\mathrm{d}}$ e um acréscimo de 2,5\% para $\mathrm{LVA}_{\mathrm{h}}$; na dose $250 \mathrm{mg}$ $\mathrm{dm}^{-3}, \mathrm{o} \mathrm{LV}_{\mathrm{d}}$ apresentou uma queda de aproximadamente $30,0 \%$, enquanto no $\mathrm{LVA}_{\mathrm{h}}$ a mesma dose aplicada não afetou a produção de grãos, ocorrendo um aumento de cerca 2,0\% em relação ao tratamento-controle. Pode-se notar, que entre as doses 250 e $500 \mathrm{mg}$ $\mathrm{dm}^{-3}$ houve um aumento da produção de grãos para os dois tipos de solos comparados ao tratamento controle. Apesar dos teores de $\mathrm{Pb}$ extraídos pelo DTPA serem bastante elevados, parece razoável admitir que o metal estivesse pouco disponível às plantas ou que suas raízes atuaram como um filtro natural, limitando a translocação deste elemento para parte aérea, fato constatado por Oliveira et al. ${ }^{31} \mathrm{em}$ estudos semelhantes realizados em plantas cultivadas em solos que receberam lodo de esgoto enriquecido com cádmio e zinco. Nesses estudos, os teores de metais pesados nas folhas e nas raízes foram superiores aos dos grãos.

Síntese e caracterização recentes de quelatos de $\mathrm{Cu}, \mathrm{Zn}, \mathrm{Cd}$ e $\mathrm{Pb}$ com DTPA mostraram tratar-se de estruturas com elevado impedimento estérico. ${ }^{32,33}$ Considerando que o DTPA se assemelha muito aos aminoácidos presentes na seiva das plantas, parece razoável atribuir a baixa translocação de metais pesados, notadamente $\mathrm{Pb}$, à formação de estruturas semelhantes nas plantas. 


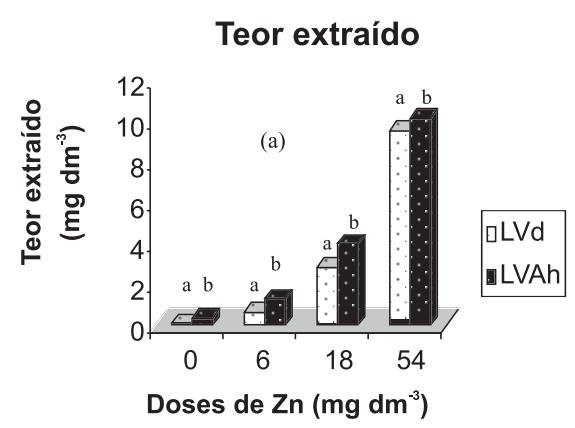

Teor extraído

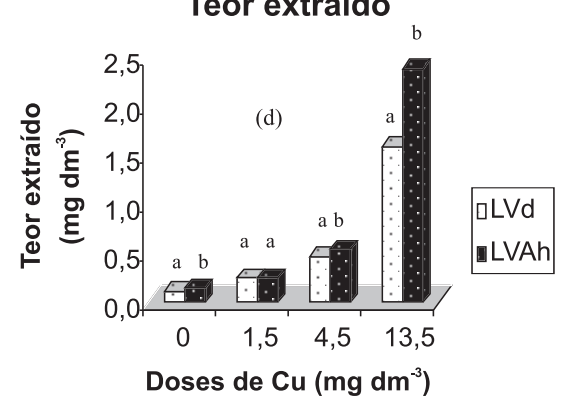

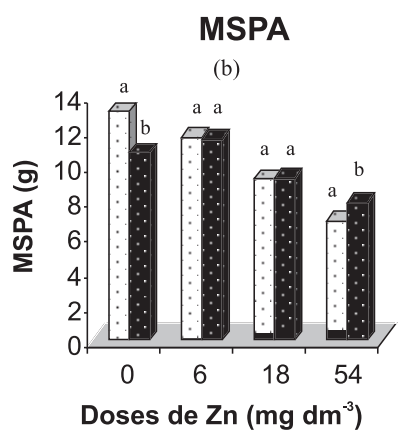

\begin{tabular}{l}
\hline LVd \\
LVAh \\
\hline
\end{tabular}

MSPA

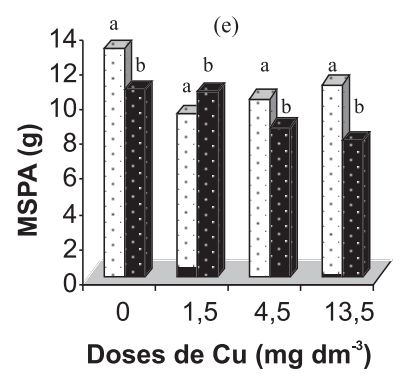

Produção de grãos

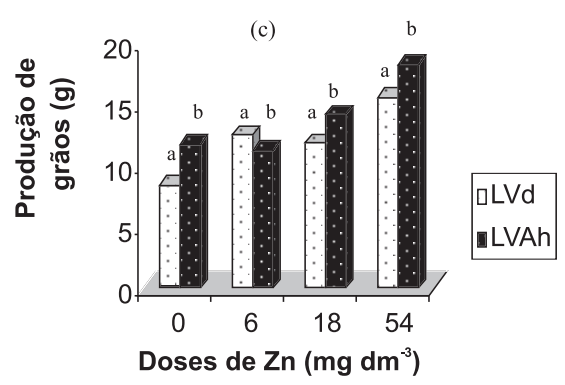

Produção de grãos

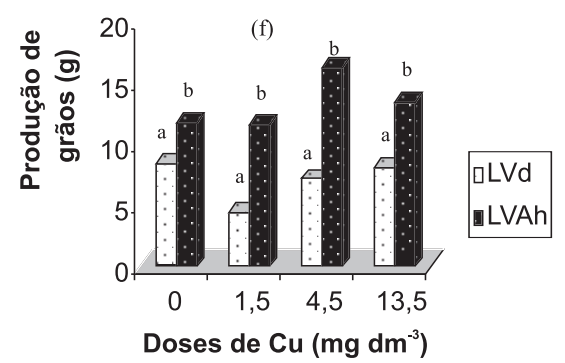

Figura 2. Teores de metais solúveis no extrator DTPA, produção de matéria seca e de grãos por plantas de feijoeiro cultivadas em dois solos, em função das doses de $\mathrm{Zn}(a, b, c)$ e Cu $(d, e, f)$ aplicadas. Médias seguidas pela mesma letra não diferem entre si pelo teste de Tukey em nível de $5 \%$ de probabilidade

\section{Zinco}

Os teores de Zn solúveis no DTPA (Figura 2a) em função das doses nos dois solos estudados mostram que houve discreta vantagem para o LVA, conforme se deduz da observação dos gráficos. Respectivamente, embora próximos, demonstram a acentuada capacidade de retenção do metal pelos dois solos, já que foram estimados a partir da dose máxima aplicada, de $54 \mathrm{mg} \mathrm{dm}^{-3}$. Esses números indicam que apenas cerca de $18,0 \%$ da maior dose foram extraídos pelo DTPA permanecendo a maior parte do $\mathrm{Zn}$ aplicado retido nos dois solos.

A retenção do $\mathrm{Zn}$ parece estar associada à matéria orgânica e à natureza da fração argila dos solos (Tabela 1). Os valores 3,1 e 2,5 $\mathrm{g} \mathrm{kg}^{-1}$ de MO para o LVA $\mathrm{h}$ o $\mathrm{LV}_{\mathrm{d}}$, respectivamente, parecem indicar, não importando o estágio de decomposição do material vegetal de origem, a presença de radicais orgânicos carboxilados, sulfurados e nitrogenados, potenciais doadores de elétrons ao $\mathrm{Zn}$ em reações ácido-base de Lewis de formação de quelatos. Estes, devido à estabilidade, permanecem adsorvidos às superfícies sólidas dos solos utilizados neste trabalho, tanto nas amostras naturais de campo (Tabela 1), quanto no material que recebeu os tratamentos experimentais e a fertilização básica para plantio (Tabela 3). Há um predomínio de espécie $\mathrm{Zn}^{2+}$ no solo desde que o $\mathrm{pH}$ seja menor que 8 , enquanto que em valores maiores prevalecem íons do tipo $[\mathrm{ZnOH}]^{+},\left[\mathrm{Zn}(\mathrm{OH})_{3}\right]^{-}$e $\left[\mathrm{Zn}(\mathrm{OH})_{4}\right]^{-6}{ }^{6}$ Quando o $\mathrm{pH}$ do solo aumenta, o $\mathrm{Zn}$ é mais adsorvido pelos óxidos e oxi-hidróxidos de $\mathrm{Fe}$ e de $\mathrm{Al}^{6}$

Esperava-se uma maior retenção de $\mathrm{Zn}$ no $\mathrm{LVA}_{\mathrm{h}}$ devido ao maior teor de MO (Tabela1), entretanto é razoável admitir ter havido uma preferência maior do Zn pelo DTPA como ligante que pelos radicais complexantes do material orgânico. A preferência parece advir do fato da molécula do complexante ser octadentada e, assim, melhor doadora de elétrons que quaisquer outros grupos funcionais da matéria orgânica.

A redução da produção de MSPA pelas plantas cultivadas no
LVA $_{\mathrm{h}}$ correspondeu a uma variação de $44 \%$. Quanto ao $\mathrm{LV}_{\mathrm{d}}$, o percentual de redução da MSPA quadrático foi de 48\%, comparando-se as doses máximas e mínima de $\mathrm{Zn}$ aplicadas (Figura 2b). Desses números parece lógico deduzir prejuízos às plantas provocados por um excesso de Zn no solo, entretanto, pode-se considerar insignificante a perda de MSPA no $\mathrm{LVA}_{\mathrm{h}}$ e ocorrendo um aumento na produção no $\mathrm{LV}_{\mathrm{d}}$ com a dose de $6 \mathrm{mg} \mathrm{dm}^{-3}$ de $\mathrm{Zn}$, valor próximo do teor ideal no solo desse elemento essencial às plantas ${ }^{25}$ (Figura $2 b$ ).

Os dados da Figura 2c, relacionando as doses crescentes de $\mathrm{Zn}$ nos solos com a produção de grãos pelo feijoeiro, confirmam que a redução da MSPA das plantas pelo incremento das doses do metal não correspondeu a uma diminuição da produção de grãos como era esperado. Pelo contrário, os ajustes matemáticos para os dois solos, muito semelhantes entre si, mostram aumento da produção de grãos, apesar da diminuição da MSPA.

\section{Cobre}

Para o cobre (Figura 2d) observa-se que ocorre um aumento dos teores extraídos pelo DTPA em função do aumento das doses aplicadas para o $\mathrm{LV}_{\mathrm{d}}$ e para o $\mathrm{LVA}_{\mathrm{h}}$. Entre o tratamento-controle e a dose 4,5 $\mathrm{mg} \mathrm{dm}^{-3}$ a extração foi muito semelhante nos dois solos, estando disponível para as plantas somente $20 \%$ na dose $1,5 \mathrm{mg}$ $\mathrm{dm}^{-3}$ e $10,5 \%$ para a dose $4,5 \mathrm{mg} \mathrm{dm} \mathrm{m}^{-3}$ em relação ao $\mathrm{LV}_{\mathrm{d}}$. No LVA, a disponibilidade do metal ficou em torno de $17,3 \%$ para dose 1,5 $\mathrm{mg} \mathrm{dm}{ }^{-3}$ e $12,0 \%$ para dose $4,5 \mathrm{mg} \mathrm{dm}^{-3}$, permanecendo o restante do $\mathrm{Cu}$ aplicado retido no solo. Como acontece com a maioria dos metais pesados, $\mathrm{pH}, \mathrm{CTC}, \mathrm{MO}$, além da composição física, química e mineralógica do solo parecem ter contribuído decididamente para a dinâmica dos metais no solo. Conforme a Tabela 1 (solo amostrado) o pH era de 6,1 para $\mathrm{LV}_{\mathrm{d}}$ e 5,4 para $\mathrm{LVA}_{\mathrm{h}}$; após fertilização básica (Tabela 3), o pH teve um acréscimo alcançando um patamar ligeiramente básico de 7,3 para o $\mathrm{LV}_{\mathrm{d}}$ e de 6,3 para o $\mathrm{LVA}_{\mathrm{h}}$.

$\mathrm{O}$ comportamento do $\mathrm{pH}$ parece concordar com as observações de Martinez e Motto, ${ }^{34}$ segundo os quais o pH em torno de 5,3 é um 
indicador de limiar de toxidez do $\mathrm{Cu}$ para as plantas. Abaixo desse valor, o $\mathrm{Cu}$ torna-se muito móvel e bastante disponível à absorção na solução do solo. Os pHs mais elevados decorrentes da fertilização básica (Tabela 3) podem ter contribuído para imobilizar o $\mathrm{Cu}$ por quelatização, fato que aparentemente explica os baixos teores extraídos pelo DTPA nos dois solos (Figura 2d).

A afinidade do $\mathrm{Cu}$ por solos, de modo geral, pode ser atribuída à sua forte tendência à hidrólise que gera complexos carregados, fortemente atraídos por superfícies de troca ou por ligantes solúveis na fase líquida dos solos. ${ }^{6}$

A CTC efetiva do $\mathrm{LV}_{\mathrm{d}}$ aumentou consideravelmente quando o solo nativo (Tabela 1) recebeu a fertilização básica (Tabela 3), passando de $0,7 \mathrm{cmol}_{\mathrm{c}} \mathrm{dm}^{-3}$ para valores em torno de $3 \mathrm{cmol}_{\mathrm{c}} \mathrm{dm}^{-3}$. A mesma comparação (Tabelas 1 e 3 ) feita para o LVA mostra que a fertilização básica pouco alterou o referido parâmetro. Portanto, parte do baixo poder extrativo do DTPA em ambos os solos parece estar ligado às suas elevadas CTCs verificadas após a fertilização básica.

A complexação do cobre pela MO do solo é mais forte entre os metais de transição divalentes, como é o caso do $\mathrm{Cu}^{2+}{ }^{2} \mathrm{~A}$ imobilidade do $\mathrm{Cu}$ normalmente é atribuída à formação de complexos orgânico-metálicos estáveis e de baixa solubilidade, ${ }^{6}$ além da complexação por substâncias orgânicas, podendo a imobilidade do $\mathrm{Cu}$ também ser devida a sua ligação com frações não-trocáveis do solo..$^{35}$

Na Figura 2e observa-se o comportamento da MSPA em função das doses de $\mathrm{Cu}$ aplicadas. No $\mathrm{LV}_{\mathrm{d}}$ a partir da dose $4,5 \mathrm{mg} \mathrm{dm}^{-3}$ houve um aumento na produção de MSPA, que pode ter ocorrido por estar o elemento pouco disponível em solução para as plantas em relação às doses aplicadas. Infere-se daí que a baixa disponibilidade, ou teor extraído pelo DTPA (dados da Figura 2d), foi suficiente para suprir as necessidades de $\mathrm{Cu}$ das plantas. Esse fato parece só ter sido válido para as doses 4,5 e $13,5 \mathrm{mg} \mathrm{dm}^{-3}$, já que na dose $1,5 \mathrm{mg} \mathrm{dm}^{-3}$ o teor disponível foi muito baixo por se tratar provavelmente de uma dose baixa. Para o LVA, em todas as doses aplicadas observou-se uma queda na produção de MSPA, ocorrendo uma variação de $27,4 \%$ comparando o tratamento-controle com a maior dose aplicada.

No LVA a partir do tratamento-controle houve um aumento da produção de grãos em todas as doses aplicadas, variando de $11,6 \mathrm{~g}$ no tratamento-controle, para $16,2 \mathrm{~g}$ na dose $4,5 \mathrm{mg} \mathrm{dm}^{-3}$, sendo maior incremento observado na produção, cerca de 45,0\%. O aumento de $15,2 \%$ verificado para a dose $13,5 \mathrm{mg} \mathrm{dm}^{-3} \mathrm{em}$ comparação ao tratamento-controle foi menor quando comparado com a produção na dose $4,5 \mathrm{mg} \mathrm{dm}^{-3}$. Com relação ao $\mathrm{LV}_{\mathrm{d}}$ houve variações distintas para as doses aplicadas, sendo que somente na dose $1,5 \mathrm{mg} \mathrm{dm}^{-3}$ ocorreu uma queda mais brusca, cerca de $48,0 \%$. A partir da dose $4,5 \mathrm{mg} \mathrm{dm}^{-3}$ houve um aumento na produção de grãos, quando comparada com a dose $1,5 \mathrm{mg} \mathrm{dm}^{-3}$, sendo que a produção foi bem próxima aos valores observados no tratamento-controle.

\section{CONCLUSÕES}

$\mathrm{Zn}, \mathrm{Cu}, \mathrm{Cd}$ e Pb extraídos por DTPA aumentam com o aumento das doses aplicadas nos solos.

Nas doses usadas, $\mathrm{Cu}, \mathrm{Zn}, \mathrm{Cd}$ e $\mathrm{Pb}$ reduzem a produção de matéria seca da parte aérea e $\mathrm{o} \mathrm{Pb}$ e $\mathrm{Zn}$ pouco afetam a produção de grãos pelo feijoeiro nos dois solos.

\section{REFERÊNCIAS}

1. Paim, L. A.; Carvalho,R.; Abreu, C. M. P.; Guerreiro, M. C.; Quim. Nova 2006, 29, 28

2. Lima, M. C.; Giacomelli, M. B.; Supp, V.; Roberge, F. D.; Barrera, P. B.; Quim. Nova 2001, 24, 734 .

3. Jesus, H. C.; Costa, E. A.; Mendonça, A. S. F.; Zandonade, E.; Quim. Nova 2004, 27, 378 .

4. Cotta, J. A. O.; Rezende, M. O. O.; Piovani, M. R.; Quim. Nova 2006, 29, 40.

5. Abdel-Hallem, A. S.; Scroon, A.; El-Bahi, S. M.; Zohny, E.; Appl. Radiat. Isot. 2001, 55, 569

6. Mc Bride, M. D.; Environmental Chemistry of soils, Oxford University: New York, 1994

7. Ferreira, M. E.; Curz, M. C. P.; Raij, B. V.; Abreu, C. A.; Micronutrientes e elementos tóxicos na agricultura, CNPq, FAPESP, Potafos: Jaboticabal, 2001.

8. Pierangeli, M. A. P.; Guilherme, L. R. G.; Curi, N.; Silva, M. L. N.; Lima, J. M.; Costa, E. T. S.; R. Bras. Ci. Solo 2005, 29, 269.

9. Moreira, F. M. S.; Siqueira, J. O.; Microbiologia e bioquímica do solo, $2^{\mathrm{a}}$ ed., UFLA: Lavras, 2006.

10. Malavolta, E.; Fertilizantes e seu Impacto ambiental, metais pesados, mitos, mistificações e fatos, Produquímica: São Paulo, 1994.

11. Kabata-Pendias, ?.; Pendias, H.; Trace elements in soils and plants, Boca Raton: Flórida, 2001.

12. Baird, C.; Quimica Ambiental, $2^{\circ}$ ed., Bookman: Porto Alegre, 2003.

13. Alloway, B. J.; Heavy metals in soils, Jonh Willey Sons: New York, 1993.

14. Campos, M. L.; Silva, F. N.; Neto, A. E. F.; Guilherme, L. R. G.; Marques, J. J.; Antunes, A. S.; R. Bras. Ci. Solo 2005, 40, 265.

15. Alcarde, J. C.; Vale, F.; R. Bras. Ci. Solo 2004, 27, 254.

16. Nascimento, C. W. A.; Barros, D. A. S.; Melo, E. E. C.; Oliveira, A. B.; $R$. Bras. Ci. Solo 2004, 27, 273.

17. Ferreira, M. E. Em ref. 7

18. Curi, N.; Marques, J. J.; Guilherme, L. R. G.; Lima, J. M.; Lopes, A. S.; Alvarez, V.; Tópicos em ciência do solo, $2^{\mathrm{a}}$ ed., UFLA: Lavras, 2002.

19. Lindsay, W. L.; Norwell, W. A.; Soil. Sci. Soc. Am. J. 1978, 42, 421.

20. Anjos, A. R. M.; Mattiazzo, M. E.; Sci. Agric. 2001, 58, 337.

21. EMBRAPA - Empresa Brasileira de Pesquisa Agropecuária; Manual de Métodos de Análise de solo, Centro Nacional de Pesquisa de Solos: Rio de Janeiro, 1997.

22. Alvarez, V.; Fonseca, V. H.; R. Bras. Ci. Solo 1990, 14, 49.

23. Quaggio, J. A.; Raij, B. V.; Malavolta, E.; Commun. Soil. Sci. Plant. Anal. 1985, 16, 245.

24. Freire, J. C.; Ribeiro, M. A. V.; Bahia, V. G.; Lopes, A. S.; Aquino, L. H.; R. Bras. Ci. Solo. 1980, 4, 5.

25. Malavolta, E.; Elementos de nutrição mineral de plantas, Ceres: São Paulo, 1980.

26. Zonta, E. P.; Machado, A. A.; Manual do SANEST: sistema de análise estatística para microcomputadores, UFP: Pelotas, 1991.

27. Krishnamurti, G. S. R.; Naidu, R.; Solid-solution equilibria of Cadmium of soils, Geoderma, 2003.

28. Seregin, L. V.; Ivanov, V. B.; Russ. J. Plant. Physiol, 2001, 48, 523

29. Pierangeli, M. A. P.; Guilherme, L. R. G.; Curi, N.; Silva, M. L. N.; Oliveira, L. R.; Lima, J. M.; R. Bras. Ci. Solo 2001, 25, 279.

30. Miller, J. E.; J. Environ. Qual. 1976, 5, 157.

31. Oliveira, C.; Sobrinho, N. M. B. A.; Marques, V. S.; Mazur, N.; R. Bras. Ci. Solo 2005, 29, 1.

32. Silva, V. L.; Carvalho, R.; Freitas, M. P.; Tormena, C. F.; Melo, W. C.; Spectrochim. Acta 2007, 68, 1197.

33. Silva, V. L.; Carvalho, R.; Freitas, M. P.; Tormena, C. F.; Melo, W. C.; Struct. Chem. 2007, 18, 605.

34. Martinez, C. E.; Motto, H. L.; Environ. Pollut. 2000, 107, 153.

35. Matos, A. T.; Fontes, M. P. F.; Jordão, C. P.; Costa, L. M.; R. Bras. Ci. Solo 1996, 20, 379. 